\title{
Bromine and iodine chemistry in a global chemistry- climate model: Description and evaluation of very short-lived oceanic sources
}

Carlos Ordóñez ${ }^{1}$, Jean-François Lamarque ${ }^{2}$, Simone Tilmes $^{2}$, Douglas E. Kinnison ${ }^{2}$, Elliot L. Atlas ${ }^{3}$, Donald R. Blake ${ }^{4}$, Gabriela Sousa Santos ${ }^{5}$, Guy Brasseur ${ }^{6}$, and Alfonso Saiz-Lopez ${ }^{1}$

${ }^{1}$ Laboratory for Atmospheric and Climate Science (CIAC), CSIC, Toledo, Spain

${ }^{2}$ Atmospheric Chemistry Division, NCAR, Boulder, CO, USA

${ }^{3}$ University of Miami, Miami, FL, USA

${ }^{4}$ University of California, Irvine, CA, USA

${ }^{5}$ Institute of Atmospheric and Climate Science, ETH, Zurich, Switzerland

${ }^{6}$ Climate Service Center, Hamburg, Germany 


\section{Chemistry of VSL halogen species in CAM-Chem}

An introduction to the tropospheric halogen chemistry scheme in CAM-Chem is given in Section 3 of the main text. That section also describes the breakdown of very shortlived (VSL) halocarbons into their products. Here we focus on the main gas phase reactions involving reactive bromine and iodine in the model, in particular those which are relevant for tropospheric ozone $\left(\mathrm{O}_{3}\right)$ depletion. We will also briefly summarise other processes of importance for the troposphere, following the review by von Glasow and Crutzen (2007). The bimolecular, termolecular and photolytic reactions involving halogen species in the model are summarised in Tables 1, 2 and 3, respectively. Information on heterogeneous reactions, Henry's Law constants and dry deposition velocities of halogen species is included in Tables 4, 5 and 6, respectively. In the following, $\mathrm{X}$ represents $\mathrm{Cl}, \mathrm{Br}$ or $\mathrm{I}$. If two halogen species are present in the same reaction then we use $\mathrm{X}$ and $\mathrm{Y}$ to design both of them.

In addition to the photochemical breakdown of halocarbons, halogen radicals $(\mathrm{X}, \mathrm{Y})$ can be formed in the troposphere by the photolysis of dihalogens $\left(\mathrm{X}_{2}\right.$ or $\left.\mathrm{XY}\right)$ or other inorganic halogen species like HOX (Table 3). They react with $\mathrm{O}_{3}$ producing halogen oxides (XO). If these oxides photolyse, both $\mathrm{O}_{3}$ and the halogen radical $\mathrm{X}$ are formed again; however they can also undergo chemical reactions that lead to net $\mathrm{O}_{3}$ destruction. One of the main $\mathrm{O}_{3}$ destruction paths includes the reaction of $\mathrm{XO}$ with $\mathrm{HO}_{2}$, which is also responsible for reducing the $\mathrm{HO}_{\mathrm{x}}\left(\mathrm{HO}_{2} / \mathrm{OH}\right)$ ratio:

$$
\begin{aligned}
& X+\mathrm{O}_{3} \rightarrow \mathrm{XO}+\mathrm{O}_{2} \\
& \mathrm{XO}+\mathrm{HO}_{2} \rightarrow \mathrm{HOX}+\mathrm{O}_{2} \\
& \mathrm{HOX}+h v \rightarrow X+\mathrm{OH}
\end{aligned}
$$

$$
\text { Net: } \mathrm{HO}_{2}+\mathrm{O}_{3} \rightarrow \mathrm{OH}+2 \mathrm{O}_{2}
$$

$\mathrm{BrO}$ and IO self-reactions are also of importance for tropospheric ozone destruction:

$$
\begin{aligned}
& 2\left(\mathrm{Br}+\mathrm{O}_{3} \rightarrow \mathrm{BrO}+\mathrm{O}_{2}\right) \\
& \mathrm{BrO}+\mathrm{BrO} \rightarrow 2 \mathrm{Br}+\mathrm{O}_{2}
\end{aligned}
$$


Net: $2 \mathrm{O}_{3} \rightarrow 3 \mathrm{O}_{2}$

$$
\begin{aligned}
& 2\left(\mathrm{I}+\mathrm{O}_{3} \rightarrow \mathrm{IO}+\mathrm{O}_{2}\right) \\
& \mathrm{IO}+\mathrm{IO} \rightarrow \mathrm{OIO}+\mathrm{I} \\
& \mathrm{OIO}+h v \rightarrow \mathrm{I}+\mathrm{O}_{2}
\end{aligned}
$$

Net: $2 \mathrm{O}_{3} \rightarrow 3 \mathrm{O}_{2}$

When two halogen oxides are present in the troposphere, cross reactions such as

$$
\begin{aligned}
& \mathrm{BrO}+\mathrm{ClO} \rightarrow \mathrm{Br}+\mathrm{Cl}+\mathrm{O}_{2} \\
& \mathrm{IO}+\mathrm{BrO} \rightarrow \mathrm{I}+\mathrm{Br}+\mathrm{O}_{2}
\end{aligned}
$$

can make $\mathrm{O}_{3}$ destruction very efficient. Photochemical box model calculations of observations in coastal Antarctica (Saiz-Lopez et al., 2007) and the tropical Atlantic Ocean (Read et al., 2008) have shown that the effect of IO and BrO together is greater than the sum of their individual contributions, because the reaction $\mathrm{IO}+\mathrm{BrO}$ increases the rate at which the halogen oxides are converted back to $\mathrm{I}$ and $\mathrm{Br}$ atoms. Other reaction paths for the $\mathrm{BrO}+\mathrm{ClO}$ reaction produce $\mathrm{OClO}(\mathrm{R} 48)$ and $\mathrm{BrCl}(\mathrm{R} 50)$, while an additional channel of the $\mathrm{IO}+\mathrm{BrO}$ reaction yields $\mathrm{OIO}(\mathrm{R} 85)$.

In very clean air $(\mathrm{NOx}<30 \mathrm{ppt})$, the gas-phase chemistry of IO is dominated by the reactions of $\mathrm{IO}$ with itself to form $\mathrm{I}_{2} \mathrm{O}_{2}$ and $\mathrm{OIO}+\mathrm{I}$, $\mathrm{OO}+\mathrm{OIO}$ to form $\mathrm{I}_{2} \mathrm{O}_{3}$, and $\mathrm{OIO}+$ OIO to form $\mathrm{I}_{2} \mathrm{O}_{4}$ (Table 1). These reactions proceed rapidly in the gas phase, with rate coefficients of $\sim 10^{10}-10^{11}$. In the current version of CAM-Chem we do not include the formation of gas phase $\mathrm{I}_{2} \mathrm{O}_{5}$ through oxidation of $\mathrm{I}_{2} \mathrm{O}_{4}$ by $\mathrm{O}_{3}$. We also assume that, with the only exception of the very rapid thermal decomposition of $\mathrm{I}_{2} \mathrm{O}_{2}\left(\mathrm{R} 73\right.$ and R74), $\mathrm{I}_{2} \mathrm{O}_{\mathrm{x}}$ $(\mathrm{x}=2,3,4)$ do not undergo photolysis or other reactions which would reduce them to IO or OIO. Hence, the rapid conversion of IO and OIO into higher order iodine oxides limits the atmospheric lifetime of both radicals in the model. 1-D model calculations strongly suggest that the photolysis of $\mathrm{I}_{2} \mathrm{O}_{2}, \mathrm{I}_{2} \mathrm{O}_{3}, \mathrm{I}_{2} \mathrm{O}_{4}$ and $\mathrm{I}_{2} \mathrm{O}_{5}$ would be required to explain the vertical distribution of IO in the Antarctic boundary layer (Saiz-Lopez et al., 2008). However further laboratory work on the photochemistry of these species is needed. 
The formation of halogen nitrates and nitrites can be important in semi-polluted environments:

$$
\begin{aligned}
& X O+\mathrm{NO}_{2}(+M) \rightarrow \mathrm{XONO}_{2}(+M) \\
& X+\mathrm{NO}_{2}(+M) \rightarrow \mathrm{XNO}_{2}(+M)
\end{aligned}
$$

Depending on conditions, these species can either photolyse

$$
\begin{aligned}
& \mathrm{XONO}_{2}+h v \rightarrow X+\mathrm{NO}_{3} \\
& \mathrm{XONO}_{2}+h v \rightarrow \mathrm{XO}+\mathrm{NO}_{2} \\
& \mathrm{XNO}_{2}+h v \rightarrow \mathrm{X}+\mathrm{NO}_{2} \\
& \mathrm{XNO}_{2}+h v \rightarrow \mathrm{XO}+\mathrm{NO}
\end{aligned}
$$

thermally decompose

$$
\mathrm{INO}_{2} \rightarrow \mathrm{I}+\mathrm{NO}_{2}
$$

undergo oxidation (see Table 1) or react on surfaces (Table 4). Some of these reactions can also lead to $\mathrm{O}_{3}$ depletion.

Sea salt is also an important source of reactive bromine and iodine in the troposphere. Typical diameters of sea salt particles are $>0.1 \mu \mathrm{m}$, which limits their relevance to the marine boundary layer due to fast removal by sedimentation and wet scavenging. In CAM-Chem, the heterogeneous recycling of halogens on sea salt aerosols is treated as follows. The uptake and subsequent hydrolysis of $\mathrm{XONO}_{2}, \mathrm{HOX}$ and $\mathrm{XNO}_{2}$ on marine aerosols produces HOX, which equilibrates between gas and aqueous phase according to its Henry's law solubility. The processing of aqueous $\mathrm{HOX}$ to $\mathrm{Br}_{2}, \mathrm{BrCl}, \mathrm{IBr}, \mathrm{ICl}$ and $\mathrm{Cl}_{2}$ via reaction with $\mathrm{Br}^{-}, \mathrm{Cl}^{-}$and $\mathrm{I}^{-}$takes only between 10 and 15 min on fresh sea-salt aerosols (McFiggans et al., 2000). Since di-halogen molecules are insoluble they are rapidly released to the gas phase. As a consequence, uptake of inorganic bromine and iodine species onto aerosols is the rate-limiting step of the process (McFiggans et al., 2000). Aged sea-salt aerosols become depleted in $\mathrm{Br}^{-}$and $\mathrm{Cl}^{-}$thus slowing down the halogen recycling on aerosols (e.g. Fickert et al., 1999; von Glasow et al., 2002). Additionally, aged particles become progressively acidified by uptake of $\mathrm{HNO}_{3}, \mathrm{H}_{2} \mathrm{SO}_{4}$ and $\mathrm{SO}_{2}$, which in turn increases the rate of processing. In CAM-Chem we do not explicitly treat the aqueous phase chemistry in the bulk of the sea salt aerosols. Instead, we assume that the limiting step for halogen recycling on sea salt aerosols is the first- 
order rate of uptake computed for a number of gaseous species using the free molecular transfer regime approximation (see Table 4). CAM-Chem simulations indicate that heterogeneous processing of bromine on sea-salt aerosol is needed to reproduce the observed $\mathrm{BrO}$ in the marine boundary layer, but the full description of all the processes which release halogens from sea-salt aerosols is out of the scope of this paper. Note that the photochemical processing of bromocarbons provides the main source of mid- and upper tropospheric reactive bromine due to their longer lifetimes relative to those of most inorganic bromine species, as already pointed out by Yang et al. (2005) and Warwick et al. (2006).

The halogen species removed in CAM-Chem by washout and scavenging in clouds are listed in Table 5 together with the corresponding Henry Law's coefficients. In addition, dry deposition to the surface occurs from the lowermost level of the model for the following reactive halogen species: $\mathrm{HCl}, \mathrm{HBr}, \mathrm{HOCl}, \mathrm{ClONO}_{2}, \mathrm{HOBr}, \mathrm{BrONO}_{2}, \mathrm{HI}$, $\mathrm{I}_{2} \mathrm{O}_{2}, \mathrm{HOI}, \mathrm{IONO}_{2}, \mathrm{INO}_{2}$ (see Table 6 of the Supplementary Material). The deposition flux of each gaseous species is calculated as the product of the deposition velocity and the concentration. 
Table 1. Halogen chemistry scheme in CAM-Chem. Bimolecular and thermal decomposition reactions.

\begin{tabular}{|c|c|c|c|}
\hline & Reactions & $\begin{array}{c}\text { Rate Constants } \\
\left(\mathrm{cm}^{3} \text { molecule }^{-1} \mathrm{~s}^{-1}\right)\end{array}$ & Notes \\
\hline $\mathrm{R} 1$. & $\mathrm{CH}_{3} \mathrm{Cl}+\mathrm{Cl} \rightarrow \mathrm{HO}_{2}+\mathrm{CO}+2 \mathrm{HCl}$ & $3.20 \times 10^{-11} \mathrm{e}^{(-1250 / \mathrm{T})}$ & 1 \\
\hline $\mathrm{R} 2$. & $\mathrm{CH}_{3} \mathrm{Cl}+\mathrm{OH} \rightarrow \mathrm{Cl}+\mathrm{H}_{2} \mathrm{O}+\mathrm{HO}_{2}$ & $2.40 \times 10^{-12} \mathrm{e}^{(-1250 / \mathrm{T})}$ & 2 \\
\hline R3. & $\mathrm{CH}_{3} \mathrm{CCl}_{3}+\mathrm{OH} \rightarrow \mathrm{H}_{2} \mathrm{O}+3 \mathrm{Cl}$ & $1.60 \times 10^{-12} \mathrm{e}^{(-1520 / \mathrm{T})}$ & 1 \\
\hline R4. & $\mathrm{HCFC} 22+\mathrm{OH} \rightarrow \mathrm{Cl}+\mathrm{H}_{2} \mathrm{O}+\mathrm{CF}_{2} \mathrm{O}$ & $4.00 \times 10^{-12} \mathrm{e}^{(-1400 / \mathrm{T})}$ & 1 \\
\hline R5. & $\mathrm{CH}_{3} \mathrm{Br}+\mathrm{OH} \rightarrow \mathrm{Br}+\mathrm{H}_{2} \mathrm{O}+\mathrm{HO}_{2}$ & $2.35 \times 10^{-12} \mathrm{e}^{(-1300 / \mathrm{T})}$ & 2 \\
\hline R6. & $\mathrm{CH}_{2} \mathrm{BrCl}+\mathrm{OH} \rightarrow \mathrm{Br}+\mathrm{Cl}$ & $2.40 \times 10^{-12} \mathrm{e}^{(-920 / \mathrm{T})}$ & 2 \\
\hline R7. & $\mathrm{CH}_{2} \mathrm{Br}_{2}+\mathrm{OH} \rightarrow 2 \mathrm{Br}$ & $2.00 \times 10^{-12} \mathrm{e}^{(-840 / \mathrm{T})}$ & 2 \\
\hline R8. & $\mathrm{CHBr}_{3}+\mathrm{OH} \rightarrow 3 \mathrm{Br}$ & $1.35 \times 10^{-12} \mathrm{e}^{(-600 / \mathrm{T})}$ & 2 \\
\hline R9. & $\mathrm{CHBrCl}_{2}+\mathrm{OH} \rightarrow \mathrm{Br}+2 \mathrm{Cl}$ & $9.00 \times 10^{-13} \mathrm{e}^{(-600 / \mathrm{T})}$ & 3 \\
\hline R10. & $\mathrm{CHBr}_{2} \mathrm{Cl}+\mathrm{OH} \rightarrow 2 \mathrm{Br}+\mathrm{Cl}$ & $9.00 \times 10^{-13} \mathrm{e}^{(-600 / \mathrm{T})}$ & 3 \\
\hline R11. & $\mathrm{OH}+\mathrm{CH}_{3} \mathrm{I} \rightarrow \mathrm{CH}_{2} \mathrm{I}+\mathrm{H}_{2} \mathrm{O}$ & $2.9 \times 10^{-12} \mathrm{e}^{(-1100 / \mathrm{T})}$ & 2 \\
\hline $\mathrm{R} 12$. & $\mathrm{OH}+\mathrm{CF}_{3} \mathrm{I} \rightarrow \mathrm{HOI}+\mathrm{CF}_{3}$ & $2.5 \times 10^{-11} \mathrm{e}^{(-2070 / \mathrm{T})}$ & 2 \\
\hline R13. & $\mathrm{Cl}+\mathrm{O}_{3} \rightarrow \mathrm{ClO}+\mathrm{O}_{2}$ & $2.30 \times 10^{-11} \mathrm{e}^{(-200 / \mathrm{T})}$ & 1 \\
\hline R14. & $\mathrm{Cl}+\mathrm{H}_{2} \rightarrow \mathrm{HCl}+\mathrm{H}$ & $3.70 \times 10^{-11} \mathrm{e}^{(-2300 / \mathrm{T})}$ & 1 \\
\hline R15. & $\mathrm{Cl}+\mathrm{H}_{2} \mathrm{O}_{2} \rightarrow \mathrm{HCl}+\mathrm{HO}_{2}$ & $1.10 \times 10^{-11} \mathrm{e}^{(-980 / \mathrm{T})}$ & 1 \\
\hline R16. & $\mathrm{Cl}+\mathrm{HO}_{2} \rightarrow \mathrm{HCl}+\mathrm{O}_{2}$ & $1.80 \times 10^{-11} \mathrm{e}^{(170 / \mathrm{T})}$ & 1 \\
\hline R17. & $\mathrm{Cl}+\mathrm{HO}_{2} \rightarrow \mathrm{OH}+\mathrm{ClO}$ & $4.10 \times 10^{-11} \mathrm{e}^{(-450 / \mathrm{T})}$ & 1 \\
\hline $\mathrm{R} 18$. & $\mathrm{Cl}+\mathrm{HCHO} \rightarrow \mathrm{HCl}+\mathrm{HO}_{2}+\mathrm{CO}$ & $8.10 \times 10^{-11} \mathrm{e}^{(-30 / \mathrm{T})}$ & 1 \\
\hline R19. & $\mathrm{Cl}+\mathrm{CH}_{4} \rightarrow \mathrm{CH}_{3} \mathrm{O}_{2}+\mathrm{HCl}$ & $9.60 \times 10^{-12} \mathrm{e}^{(-1360 / \mathrm{T})}$ & 1 \\
\hline $\mathrm{R} 20$. & $\mathrm{ClO}+\mathrm{O}\left({ }^{3} \mathrm{P}\right) \rightarrow \mathrm{Cl}+\mathrm{O}_{2}$ & $3.00 \times 10^{-11} \mathrm{e}^{(+70 / \mathrm{T})}$ & 1 \\
\hline $\mathrm{R} 21$. & $\mathrm{ClO}+\mathrm{OH} \rightarrow \mathrm{Cl}+\mathrm{HO}_{2}$ & $7.40 \times 10^{-12} \mathrm{e}^{(270 / \mathrm{T})}$ & 1 \\
\hline $\mathrm{R} 22$. & $\mathrm{ClO}+\mathrm{OH} \rightarrow \mathrm{HCl}+\mathrm{O}_{2}$ & $6.0 \times 10^{-13} \mathrm{e}^{(230 / \mathrm{T})}$ & 1 \\
\hline R23. & $\mathrm{ClO}+\mathrm{HO}_{2} \rightarrow \mathrm{O}_{2}+\mathrm{HOCl}$ & $2.7 \times 10^{-12} \mathrm{e}^{(220 / \mathrm{T})}$ & 1 \\
\hline $\mathrm{R} 24$. & $\mathrm{ClO}+\mathrm{NO} \rightarrow \mathrm{NO}_{2}+\mathrm{Cl}$ & $6.40 \times 10^{-12} \mathrm{e}^{(290 / \mathrm{T})}$ & 1 \\
\hline $\mathrm{R} 25$. & $\mathrm{ClO}+\mathrm{ClO} \rightarrow 2 \mathrm{Cl}+\mathrm{O}_{2}$ & $3.00 \times 10^{-11} \mathrm{e}^{(-2450 / \mathrm{T})}$ & 1 \\
\hline R26. & $\mathrm{ClO}+\mathrm{ClO} \rightarrow \mathrm{Cl}_{2}+\mathrm{O}_{2}$ & $1.00 \times 10^{-12} \mathrm{e}^{(-1590 / \mathrm{T})}$ & 1 \\
\hline R27. & $\mathrm{ClO}+\mathrm{ClO} \rightarrow \mathrm{Cl}+\mathrm{OClO}$ & $3.50 \times 10^{-13} \mathrm{e}^{(-1370 / \mathrm{T})}$ & 1 \\
\hline $\mathrm{R} 28$. & $\mathrm{HCl}+\mathrm{OH} \rightarrow \mathrm{H}_{2} \mathrm{O}+\mathrm{Cl}$ & $2.60 \times 10^{-12} \mathrm{e}^{(-350 / \mathrm{T})}$ & 1 \\
\hline R29. & $\mathrm{HCl}+\mathrm{O}\left({ }^{3} \mathrm{P}\right) \rightarrow \mathrm{Cl}+\mathrm{OH}$ & $1.00 \times 10^{-11} \mathrm{e}^{(-3300 / \mathrm{T})}$ & 1 \\
\hline $\mathrm{R} 30$. & $\mathrm{HOCl}+\mathrm{O}\left({ }^{3} \mathrm{P}\right) \rightarrow \mathrm{ClO}+\mathrm{OH}$ & $1.70 \times 10^{-13}$ & 1 \\
\hline R31. & $\mathrm{HOCl}+\mathrm{Cl} \rightarrow \mathrm{HCl}+\mathrm{ClO}$ & $2.50 \times 10^{-12} \mathrm{e}^{(-130 / \mathrm{T})}$ & 1 \\
\hline $\mathrm{R} 32$. & $\mathrm{HOCl}+\mathrm{OH} \rightarrow \mathrm{H}_{2} \mathrm{O}+\mathrm{ClO}$ & $3.00 \times 10^{-12} \mathrm{e}^{(-500 / \mathrm{T})}$ & 1 \\
\hline R33. & $\mathrm{ClONO}_{2}+\mathrm{O}\left({ }^{3} \mathrm{P}\right) \rightarrow \mathrm{ClO}+\mathrm{NO}_{3}$ & $2.90 \times 10^{-12} \mathrm{e}^{(-800 / \mathrm{T})}$ & 1 \\
\hline R34. & $\mathrm{ClONO}_{2}+\mathrm{OH} \rightarrow \mathrm{HOCl}+\mathrm{NO}_{3}$ & $1.20 \times 10^{-12} \mathrm{e}^{(-330 / \mathrm{T})}$ & 1 \\
\hline R35. & $\mathrm{ClONO}_{2}+\mathrm{Cl} \rightarrow \mathrm{Cl}_{2}+\mathrm{NO}_{3}$ & $6.50 \times 10^{-12} \mathrm{e}^{(135 / \mathrm{T})}$ & 1 \\
\hline R36. & $\mathrm{ClNO}_{2}+\mathrm{OH} \rightarrow \mathrm{HOCl}+\mathrm{NO}_{2}$ & $2.40 \times 10^{-12} \mathrm{e}^{(-1250 / \mathrm{T})}$ & 1 \\
\hline R37. & $\mathrm{Cl}_{2}+\mathrm{OH} \rightarrow \mathrm{HOCl}+\mathrm{Cl}$ & $1.4 \times 10^{-12} \mathrm{e}^{(-900 / \mathrm{T})}$ & 1 \\
\hline R38. & $\mathrm{Br}+\mathrm{O}_{3} \rightarrow \mathrm{BrO}+\mathrm{O}_{2}$ & $1.7 \times 10^{-11} \mathrm{e}^{(-800 / \mathrm{T})}$ & 1 \\
\hline R39. & $\mathrm{Br}+\mathrm{HO}_{2} \rightarrow \mathrm{HBr}+\mathrm{O}_{2}$ & $1.5 \times 10^{-11} \mathrm{e}^{(-600 / \mathrm{T})}$ & 1 \\
\hline $\mathrm{R} 40$. & $\mathrm{Br}+\mathrm{HCHO} \rightarrow \mathrm{HBr}+\mathrm{HO} 2+\mathrm{CO}$ & $1.7 \times 10^{-11} \mathrm{e}^{(-800 / \mathrm{T})}$ & 2 \\
\hline R41. & $\mathrm{Br}+\mathrm{CH}_{3} \mathrm{CHO} \rightarrow \mathrm{HBr}+\mathrm{CH}_{3} \mathrm{CO}_{3}$ & $1.3 \times 10^{-11} \mathrm{e}^{(-360 / \mathrm{T})}$ & 11 \\
\hline $\mathrm{R} 42$. & $\mathrm{Br}+\mathrm{NO}_{3} \rightarrow \mathrm{BrO}+\mathrm{NO}_{2}$ & $1.6 \times 10^{-11}$ & 2 \\
\hline R43. & $\mathrm{Br}+\mathrm{OH} \rightarrow \mathrm{HOBr}$ & $4.2 \times 10^{-11}$ & 11 \\
\hline
\end{tabular}




\begin{tabular}{|c|c|c|}
\hline R44. & $\mathrm{BrO}+\mathrm{O}\left({ }^{3} \mathrm{P}\right) \rightarrow \mathrm{Br}+\mathrm{O}_{2}$ & $1.9 \times 10^{-11} \mathrm{e}^{(230 / \mathrm{T})}$ \\
\hline $\mathrm{R} 45$. & $\mathrm{BrO}+\mathrm{OH} \rightarrow \mathrm{Br}+\mathrm{HO}_{2}$ & $7.5 \times 10^{-11}$ \\
\hline R46. & $\mathrm{BrO}+\mathrm{HO}_{2} \rightarrow \mathrm{HOBr}+\mathrm{O}_{2}$ & $3.4 \times 10^{-12} \mathrm{e}^{(540 / \mathrm{T})}$ \\
\hline R47. & $\mathrm{BrO}+\mathrm{NO} \rightarrow \mathrm{Br}+\mathrm{NO}_{2}$ & $8.8 \times 10^{-12} \mathrm{e}^{(260 / \mathrm{T})}$ \\
\hline R48. & $\mathrm{BrO}+\mathrm{ClO} \rightarrow \mathrm{Br}+\mathrm{OClO}$ & $9.5 \times 10^{-13} \mathrm{e}^{(550 / \mathrm{T})}$ \\
\hline R49. & $\mathrm{BrO}+\mathrm{ClO} \rightarrow \mathrm{Br}+\mathrm{Cl}+\mathrm{O}_{2}$ & $2.3 \times 10^{-12} \mathrm{e}^{(260 / \mathrm{T})}$ \\
\hline R50. & $\mathrm{BrO}+\mathrm{ClO} \rightarrow \mathrm{BrCl}+\mathrm{O}_{2}$ & $4.1 \times 10^{-13} \mathrm{e}^{(290 / \mathrm{T})}$ \\
\hline R51. & $\mathrm{BrO}+\mathrm{CH}_{3} \mathrm{O}_{2} \rightarrow \mathrm{Br}+\mathrm{HCHO}+\mathrm{HO}_{2}$ & $1.6 \times 10^{-12}$ \\
\hline R52. & $\mathrm{BrO}+\mathrm{CH}_{3} \mathrm{O}_{2} \rightarrow \mathrm{HOBr}+\mathrm{HCHO}$ & $4.1 \times 10^{-12}$ \\
\hline R53. & $\mathrm{BrO}+\mathrm{CH}_{3} \mathrm{CO}_{3} \rightarrow \mathrm{Br}+\mathrm{CH}_{3} \mathrm{O}_{2}$ & $1.7 \times 10^{-12}$ \\
\hline R54. & $\mathrm{BrO}+\mathrm{BrO} \rightarrow 2 \mathrm{Br}+\mathrm{O}_{2}$ & $1.5 \times 10^{-12} \mathrm{e}^{(230 / \mathrm{T})}$ \\
\hline R55. & $\mathrm{HBr}+\mathrm{OH} \rightarrow \mathrm{Br}+\mathrm{H}_{2} \mathrm{O}$ & 5.5. $\times 10^{-12} \mathrm{e}^{(200 / \mathrm{T})}$ \\
\hline R56. & $\mathrm{HBr}+\mathrm{O}\left({ }^{3} \mathrm{P}\right) \rightarrow \mathrm{OH}+\mathrm{Br}$ & $5.8 \times 10^{-12} \mathrm{e}^{(-1500 / \mathrm{T})}$ \\
\hline R57. & $\mathrm{HBr}+\mathrm{O}\left({ }^{1} \mathrm{D}\right) \rightarrow 0.2 \mathrm{O}+0.2 \mathrm{HBr}+0.8 \mathrm{OH}+0.8 \mathrm{Br}$ & $1.5 \times 10^{-10}$ \\
\hline R58. & $\mathrm{Br}_{2}+\mathrm{OH} \rightarrow \mathrm{HOBr}+\mathrm{Br}$ & $2.1 \times 10^{-11} \mathrm{e}^{(240 / \mathrm{T})}$ \\
\hline R59. & $\mathrm{BrONO}_{2}+\mathrm{O}\left({ }^{3} \mathrm{P}\right) \rightarrow \mathrm{BrO}+\mathrm{NO}_{3}$ & $1.91 \times 10^{-11} \mathrm{e}^{(215 / \mathrm{T})}$ \\
\hline R60. & $\mathrm{BrONO}_{2}+\mathrm{Br} \rightarrow \mathrm{Br}_{2}+\mathrm{NO}_{3}$ & $1.78 \times 10^{-11} \mathrm{e}^{(365 / \mathrm{T})}$ \\
\hline R61. & $\mathrm{BrONO}_{2}+\mathrm{Cl} \rightarrow \mathrm{BrCl}+\mathrm{NO}_{3}$ & $6.28 \times 10^{-11} \mathrm{e}^{(215 / \mathrm{T})}$ \\
\hline R62. & $\mathrm{BrO}+\mathrm{DMS} \rightarrow \mathrm{Br}+\mathrm{DMSO}$ & $1.4 \times 10^{-14} \mathrm{e}^{(950 / \mathrm{T})}$ \\
\hline R63. & $\mathrm{I}_{2}+\mathrm{O}_{3} \rightarrow \mathrm{IO}+\mathrm{I}+\mathrm{O}_{2}$ & $3.8 \times 10^{-18}$ \\
\hline R64. & $\mathrm{I}_{2}+\mathrm{O}_{3} \rightarrow \mathrm{OIO}+\mathrm{IO}$ & $3.8 \times 10^{-18}$ \\
\hline R65. & $\mathrm{I}+\mathrm{O}_{3} \rightarrow \mathrm{IO}+\mathrm{O}_{2}$ & $2.1 \times 10^{-11} \mathrm{e}^{(-830 / \mathrm{T})}$ \\
\hline R66. & $\mathrm{I}+\mathrm{HO}_{2} \rightarrow \mathrm{HI}+\mathrm{O}_{2}$ & $1.5 \times 10^{-11} \mathrm{e}^{(-1090 / \mathrm{T})}$ \\
\hline R67. & $\mathrm{IO}+\mathrm{NO} \rightarrow \mathrm{I}+\mathrm{NO}_{2}$ & $7.15 \times 10^{-12} \mathrm{e}^{(300 / \mathrm{T})}$ \\
\hline R68. & $\mathrm{IO}+\mathrm{HO}_{2} \rightarrow \mathrm{HOI}+\mathrm{O}_{2}$ & $1.4 \times 10^{-11} \mathrm{e}^{(540 / \mathrm{T})}$ \\
\hline R69. & $\mathrm{IO}+\mathrm{IO} \rightarrow \mathrm{OIO}+\mathrm{I}$ & $1.6 \times 10^{-11} \mathrm{e}^{(180 / \mathrm{T})}$ \\
\hline R70. & $\mathrm{IO}+\mathrm{IO} \rightarrow \mathrm{I}_{2} \mathrm{O}_{2}$ & $3.7 \times 10^{-11} \mathrm{e}^{(180 / \mathrm{T})}$ \\
\hline R71. & $\mathrm{IO}+\mathrm{OIO} \rightarrow \mathrm{I}_{2} \mathrm{O}_{3}$ & $5.0 \times 10^{-11}$ \\
\hline $\mathrm{R} 72$. & $\mathrm{OIO}+\mathrm{OIO} \rightarrow \mathrm{I}_{2} \mathrm{O}_{4}$ & $1.5 \times 10^{-10}$ \\
\hline R73. & $\mathrm{I}_{2} \mathrm{O}_{2} \rightarrow \mathrm{OIO}+\mathrm{I}$ & $2.5 \times 10^{14} \mathrm{e}^{(-9770 / \mathrm{T})}$ \\
\hline R74. & $\mathrm{I}_{2} \mathrm{O}_{2} \rightarrow \mathrm{IO}+\mathrm{IO}$ & $1 \times 10^{12} \mathrm{e}^{(-9770 / \mathrm{T})}$ \\
\hline R75. & $\mathrm{I}_{2}+\mathrm{OH} \rightarrow \mathrm{HOI}+\mathrm{I}$ & $1.8 \times 10^{-10}$ \\
\hline R76. & $\mathrm{I}_{2}+\mathrm{NO}_{3} \rightarrow \mathrm{IO}+\mathrm{INO}_{2}$ & $1.5 \times 10^{-12}$ \\
\hline R77. & $\mathrm{I}+\mathrm{NO}_{3} \rightarrow \mathrm{IO}+\mathrm{NO}_{2}$ & $4.5 \times 10^{-10}$ \\
\hline R78. & $\mathrm{OH}+\mathrm{HI} \rightarrow \mathrm{I}+\mathrm{H}_{2} \mathrm{O}$ & $3.0 \times 10^{-11}$ \\
\hline R79. & $\mathrm{HOI}+\mathrm{OH} \rightarrow \mathrm{IO}+\mathrm{H}_{2} \mathrm{O}$ & $2.0 \times 10^{-13}$ \\
\hline R80. & $\mathrm{IO}+\mathrm{DMS} \rightarrow \mathrm{DMSO}+\mathrm{I}$ & $1.2 \times 10^{-14}$ \\
\hline R81. & $\mathrm{INO}_{2} \rightarrow \mathrm{I}+\mathrm{NO}_{2}$ & $(2.4 / 0.005) \times 2.07 \times 10^{15} \mathrm{e}^{(-11859 / \mathrm{T})}$ \\
\hline R82. & $\mathrm{INO}+\mathrm{INO} \rightarrow \mathrm{I}_{2}+2 \mathrm{NO}$ & $8.4 \times 10^{-11} \mathrm{e}^{(-2620 / \mathrm{T})}$ \\
\hline R83. & $\mathrm{INO}_{2}+\mathrm{INO}_{2} \rightarrow \mathrm{I}_{2}+2 \mathrm{NO}_{2}$ & $2.9 \times 10^{-11} \mathrm{e}^{(-2600 / \mathrm{T})}$ \\
\hline R84. & $\mathrm{IO}+\mathrm{BrO} \rightarrow \mathrm{Br}+\mathrm{I}+\mathrm{O}_{2}$ & $0.30 \times 10^{-11} \mathrm{e}^{(510 / \mathrm{T})}$ \\
\hline R85. & $\mathrm{IO}+\mathrm{BrO} \rightarrow \mathrm{Br}+\mathrm{OIO}$ & $1.20 \times 10^{-11} \mathrm{e}^{(510 / \mathrm{T})}$ \\
\hline R86. & $\mathrm{I}+\mathrm{BrO} \rightarrow \mathrm{IO}+\mathrm{Br}$ & $1.2 \times 10^{-11}$ \\
\hline R87. & $\mathrm{IO}+\mathrm{ClO} \rightarrow \mathrm{I}+\mathrm{OClO}$ & $5.1 \times 10^{-12} \mathrm{e}^{(280 / \mathrm{T})}$ \\
\hline
\end{tabular}

${ }^{1}$ JPL 02-25 (Sander et al., 2003); ${ }^{2}$ JPL 06-02 (Sander et al., 2006); ${ }^{3}$ estimated (see main text of the paper); ${ }^{4}$ Aranda et al. (1997); ${ }^{5}$ NIST (2010); ${ }^{6}$ Atkinson et al. (2007b); ${ }^{7}$ Gómez Martín et al. (2007); ${ }^{8}$ calculated using RRKM theory; ${ }^{9}$ Chambers et al. (1992); ${ }^{10}$ McFiggans et al. (2000); ${ }^{11}$ Estimated. 
Table 2. Halogen chemistry scheme in CAM-Chem. Termolecular reactions.

\begin{tabular}{|c|c|c|}
\hline & Termolecular reactions & $\begin{array}{l}\mathrm{k}=\left(\mathrm{k}_{0}[\mathrm{M}] /\left(1+\mathrm{k}_{0}[\mathrm{M}] / \mathrm{k}_{\infty}\right)\right) \times \mathrm{F}_{\mathrm{c}}{ }^{\mathrm{n}} \\
\mathrm{n}=\left\{1+\left(\log _{10}\left(\mathrm{k}_{0}[\mathrm{M}] / \mathrm{k}_{\infty}\right)\right)^{2}\right\}^{-1} \\
\mathrm{~F}_{\mathrm{c}}=\mathbf{0 . 6}\end{array}$ \\
\hline \multirow{2}{*}{\multicolumn{2}{|c|}{ T1. $\mathrm{ClO}+\mathrm{NO}_{2}(+\mathrm{M}) \rightarrow \mathrm{ClONO}_{2}(+\mathrm{M})$}} & $\mathrm{k}_{0}=1.8 \times 10^{-31} \times(\mathrm{T} / 300)^{-3.4}$ \\
\hline & & $\mathrm{k}_{\infty}=1.5 \times 10^{-11} \times(\mathrm{T} / 300)^{-1.9}$ \\
\hline \multirow{2}{*}{\multicolumn{2}{|c|}{ T2. $\mathrm{Cl}+\mathrm{NO}_{2}(+\mathrm{M}) \rightarrow \mathrm{ClNO}_{2}(+\mathrm{M})$}} & $\mathrm{k}_{0}=1.8 \times 10^{-31} \times(\mathrm{T} / 300)^{-2}$ \\
\hline & & $\mathrm{k}_{\infty}=1.0 \times 10^{-10} \times(\mathrm{T} / 300)^{-1}$ \\
\hline \multirow{2}{*}{\multicolumn{2}{|c|}{ T3. $\mathrm{ClO}+\mathrm{ClO}(+\mathrm{M}) \rightarrow \mathrm{Cl}_{2} \mathrm{O}_{2}(+\mathrm{M})$}} & $\mathrm{k}_{0}=1.6 \times 10^{-32} \times(\mathrm{T} / 300)^{-4.5}$ \\
\hline & & $\mathrm{k}_{\infty}=2.0 \times 10^{-12} \times(\mathrm{T} / 300)^{-2.4}$ \\
\hline \multirow{2}{*}{\multicolumn{2}{|c|}{ T4. $\mathrm{BrO}+\mathrm{NO}_{2}(+\mathrm{M}) \rightarrow \mathrm{BrONO}_{2}(+\mathrm{M})$}} & $\mathrm{k}_{0}=5.2 \times 10^{-31} \times(\mathrm{T} / 300)^{-3.2}$ \\
\hline & & $\mathrm{k}_{\infty}=6.9 \times 10^{-12} \times(\mathrm{T} / 300)^{-2.9}$ \\
\hline \multirow{2}{*}{\multicolumn{2}{|c|}{ T5. $\mathrm{Br}+\mathrm{NO}_{2}(+\mathrm{M}) \rightarrow \mathrm{BrNO}_{2}(+\mathrm{M})$}} & $\mathrm{k}_{0}=4.2 \times 10^{-31} \times(\mathrm{T} / 300)^{-2.4}$ \\
\hline & & $\mathrm{k}_{\infty}=2.7 \times 10^{-11}$ \\
\hline \multirow{2}{*}{\multicolumn{2}{|c|}{ T6. $\quad \mathrm{I}+\mathrm{NO}_{2}(+\mathrm{M}) \rightarrow \mathrm{INO}_{2}(+\mathrm{M})$}} & $\mathrm{k}_{0}=3 \times 10^{-31} \times(\mathrm{T} / 300)^{-1}$ \\
\hline & & $\mathrm{k}_{\infty}=6.6 \times 10^{-11}$ \\
\hline \multirow{2}{*}{\multicolumn{2}{|c|}{ T7. $\mathrm{IO}+\mathrm{NO}_{2}(+\mathrm{M}) \rightarrow \mathrm{IONO}_{2}(+\mathrm{M})$}} & $\mathrm{k}_{0}=6.5 \times 10^{-31} \times(\mathrm{T} / 300)^{-3.5}$ \\
\hline & & $\mathrm{k}_{\infty}=7.6 \times 10^{-12} \times(\mathrm{T} / 300)^{-1.5}$ \\
\hline \multirow{2}{*}{\multicolumn{2}{|c|}{ T8. $\quad \mathrm{I}+\mathrm{NO}(+\mathrm{M}) \rightarrow \mathrm{INO}(+\mathrm{M})$}} & $\mathrm{k}_{0}=1.8 \times 10^{-32} \mathrm{x}(\mathrm{T} / 300)^{-1}$ \\
\hline & & $\mathrm{k}_{\infty}=1.7 \times 10^{-11}$ \\
\hline T9. & $\mathrm{Cl}_{2} \mathrm{O}_{2}+\mathrm{M} \rightarrow \mathrm{ClO}+\mathrm{ClO}+\mathrm{M}$ & $\mathrm{K}_{\mathrm{T} 9}=\mathrm{K}_{\mathrm{T} 3} /\left[9.3 \times 10^{-28} \mathrm{xe}^{(8835 / \mathrm{T})}\right]$ \\
\hline
\end{tabular}

$\mathrm{k}_{0}$ is given in $\mathrm{cm}^{6}$ molecule $\mathrm{s}^{-1}$

$\mathrm{k}_{\infty}$ is given in $\mathrm{cm}^{3}$ molecule ${ }^{-1} \mathrm{~s}^{-1}$

All expressions taken from JPL 06-02 (Sander et al., 2006). 
Table 3. Photochemical reactions for halogen species in CAM-Chem.

\begin{tabular}{|c|c|}
\hline & Photochemical reactions of halogen species \\
\hline J1. & $\mathrm{CH}_{3} \mathrm{Cl}+h v \rightarrow \mathrm{Cl}+\mathrm{CH}_{3} \mathrm{O}_{2}$ \\
\hline $\mathrm{J} 2$. & $\mathrm{CCl}_{4}+h v \rightarrow 4 \mathrm{Cl}$ \\
\hline $\mathrm{J} 3$. & $\mathrm{CH}_{3} \mathrm{CCl}_{3}+h v \rightarrow 3 \mathrm{Cl}$ \\
\hline J4. & $\mathrm{CFC} 11+h v \rightarrow 3 \mathrm{Cl}$ \\
\hline $\mathrm{J} 5$. & $\mathrm{CFC} 12+h v \rightarrow 2 \mathrm{Cl}$ \\
\hline J6. & $\mathrm{CFC} 113+h v \rightarrow 3 \mathrm{Cl}$ \\
\hline $\mathrm{J} 7$. & $\mathrm{HCFC} 22+h v \rightarrow \mathrm{Cl}$ \\
\hline $\mathrm{J} 8$. & $\mathrm{Cl}_{2}+h v \rightarrow 2 \mathrm{Cl}$ \\
\hline J9. & $\mathrm{OClO}+h v \rightarrow \mathrm{O}+\mathrm{ClO}$ \\
\hline $\mathrm{J} 10$. & $\mathrm{Cl}_{2} \mathrm{O}_{2}+h v \rightarrow 2 \mathrm{Cl}$ \\
\hline $\mathrm{J} 11$. & $\mathrm{HOCl}+h v \rightarrow \mathrm{OH}+\mathrm{Cl}$ \\
\hline $\mathrm{J} 12$. & $\mathrm{HCl}+h v \rightarrow \mathrm{H}+\mathrm{Cl}$ \\
\hline J13. & $\mathrm{ClONO}_{2}+h v \rightarrow \mathrm{Cl}+\mathrm{NO}_{3}$ \\
\hline $\mathrm{J} 14$. & $\mathrm{ClONO}_{2}+h v \rightarrow \mathrm{ClO}+\mathrm{NO}_{2}$ \\
\hline $\mathrm{J} 15$ & $\mathrm{CH}_{3} \mathrm{Br}+h v \rightarrow \mathrm{Br}+\mathrm{CH}_{3} \mathrm{O}_{2}$ \\
\hline J16. & $\mathrm{CF}_{3} \mathrm{Br}+h v \rightarrow \mathrm{Br}$ \\
\hline J17. & $\mathrm{CF}_{2} \mathrm{ClBr}+h v \rightarrow \mathrm{Br}+\mathrm{Cl}$ \\
\hline $\mathrm{J} 18$. & $\mathrm{CHBr}_{3}+h v \rightarrow 3 \mathrm{Br}$ \\
\hline J19. & $\mathrm{CH}_{2} \mathrm{Br}_{2}+h v \rightarrow 2 \mathrm{Br}$ \\
\hline $\mathrm{J} 20$. & $\mathrm{CH}_{2} \mathrm{BrCl}+h v \rightarrow \mathrm{Br}+\mathrm{Cl}$ \\
\hline $\mathrm{J} 21$. & $\mathrm{CHBr}_{2} \mathrm{Cl}+h v \rightarrow 2 \mathrm{Br}+\mathrm{Cl}$ \\
\hline $\mathrm{J} 22$. & $\mathrm{CHBrCl}_{2}+h v \rightarrow \mathrm{Br}+2 \mathrm{Cl}$ \\
\hline $\mathrm{J} 23$. & $\mathrm{BrCl}+h v \rightarrow \mathrm{Br}+\mathrm{Cl}$ \\
\hline J24. & $\mathrm{BrO}+h v \rightarrow \mathrm{Br}+\mathrm{O}$ \\
\hline $\mathrm{J} 25$. & $\mathrm{HOBr}+h v \rightarrow \mathrm{Br}+\mathrm{OH}$ \\
\hline J26. & $\mathrm{BrONO}_{2}+h v \rightarrow \mathrm{Br}+\mathrm{NO}_{3}$ \\
\hline J27. & $\mathrm{BrONO}_{2}+h v \rightarrow \mathrm{BrO}+\mathrm{NO} 2$ \\
\hline J28. & $\mathrm{BrNO}_{2}+h v \rightarrow \mathrm{Br}+\mathrm{NO}_{2}$ \\
\hline J29. & $\mathrm{BrNO}_{2}+h v \rightarrow \mathrm{BrO}+\mathrm{NO}$ \\
\hline $\mathrm{J} 30$. & $\mathrm{CH}_{3} \mathrm{I}+h v \rightarrow \mathrm{CH}_{3}+\mathrm{I}$ \\
\hline $\mathrm{J} 31$. & $\mathrm{CH}_{2} \mathrm{I}_{2}+h v \rightarrow \mathrm{CH}_{2}+2 \mathrm{I}$ \\
\hline $\mathrm{J} 32$. & $\mathrm{CH}_{2} \mathrm{IBr}+h v \rightarrow \mathrm{CH}_{2} \mathrm{Br}+\mathrm{I}$ \\
\hline J33. & $\mathrm{CH}_{2} \mathrm{ICl}+h v \rightarrow \mathrm{CH}_{2} \mathrm{Cl}+\mathrm{I}$ \\
\hline J34. & $\mathrm{I}_{2}+h v \rightarrow 2 \mathrm{I}$ \\
\hline J35. & $\mathrm{IO}+h v \rightarrow \mathrm{I}+\mathrm{O}$ \\
\hline J36. & $\mathrm{OIO}+h v \rightarrow \mathrm{I}+\mathrm{O}_{2}$ \\
\hline J37. & $\mathrm{INO}+h v \rightarrow \mathrm{I}+\mathrm{NO}$ \\
\hline J38. & $\mathrm{INO}_{2}+h v \rightarrow \mathrm{I}+\mathrm{NO}_{2}$ \\
\hline J39. & $\mathrm{INO}_{2}+h v \rightarrow \mathrm{IO}+\mathrm{NO}$ \\
\hline J40. & $\mathrm{IONO}_{2}+h v \rightarrow \mathrm{I}+\mathrm{NO}_{3}$ \\
\hline J41. & $\mathrm{HOI}+h v \rightarrow \mathrm{I}+\mathrm{OH}$ \\
\hline J42. & $\mathrm{IBr}+h v \rightarrow \mathrm{I}+\mathrm{Br}$ \\
\hline J43. & $\mathrm{ICl}+h v \rightarrow \mathrm{I}+\mathrm{Cl}$ \\
\hline
\end{tabular}

Photolysis rates are computed online from absorption cross-sections and quantum yields reported in Atkinson et al. (2000, 2007a) and JPL 06-02 (Sander et al., 2006), and the actinic flux calculation in CAM-Chem. 
Table 4. Heterogeneous reactions involving halogens in CAM-Chem.

\begin{tabular}{|c|c|c|}
\hline & Reactions & Comments \\
\hline & \multicolumn{2}{|c|}{ Sulfate aerosol reactions } \\
\hline H1. & $\mathrm{N}_{2} \mathrm{O}_{5}+\mathrm{H}_{2} \mathrm{O} \rightarrow 2 \mathrm{HNO}_{3}$ & $*$ \\
\hline $\mathrm{H} 2$. & $\mathrm{ClONO}_{2}+\mathrm{H}_{2} \mathrm{O} \rightarrow \mathrm{HOCl}+\mathrm{HNO}_{3}$ & $*$ \\
\hline H3. & $\mathrm{BrONO}_{2}+\mathrm{H}_{2} \mathrm{O} \rightarrow \mathrm{HOBr}+\mathrm{HNO}_{3}$ & $*$ \\
\hline H4. & $\mathrm{ClONO}_{2}+\mathrm{HCl} \rightarrow \mathrm{Cl}_{2}+\mathrm{HNO}_{3}$ & $*$ \\
\hline H5. & $\mathrm{HOCl}+\mathrm{HCl} \rightarrow \mathrm{Cl}_{2}+\mathrm{H}_{2} \mathrm{O}$ & $*$ \\
\hline \multirow[t]{2}{*}{ H6. } & $\mathrm{HOBr}+\mathrm{HCl} \rightarrow \mathrm{BrCl}+\mathrm{H}_{2} \mathrm{O}$ & $*$ \\
\hline & \multicolumn{2}{|c|}{ Nitric acid tri-hydrate reactions } \\
\hline H7. & $\mathrm{N}_{2} \mathrm{O}_{5}+\mathrm{H}_{2} \mathrm{O} \rightarrow 2 \mathrm{HNO}_{3}$ & * \\
\hline H8. & $\mathrm{ClONO}_{2}+\mathrm{H}_{2} \mathrm{O} \rightarrow \mathrm{HOCl}+\mathrm{HNO}_{3}$ & $*$ \\
\hline H9. & $\mathrm{ClONO}_{2}+\mathrm{HCl} \rightarrow \mathrm{Cl}_{2}+\mathrm{HNO}_{3}$ & $*$ \\
\hline H10. & $\mathrm{HOCl}+\mathrm{HCl} \rightarrow \mathrm{Cl}_{2}+\mathrm{H}_{2} \mathrm{O}$ & $*$ \\
\hline \multirow[t]{2}{*}{ H11. } & $\mathrm{BrONO}_{2}+\mathrm{H}_{2} \mathrm{O} \rightarrow \mathrm{HOBr}+\mathrm{HNO}_{3}$ & $*$ \\
\hline & \multicolumn{2}{|c|}{ Water-ice aerosol reactions } \\
\hline H12. & $\mathrm{N}_{2} \mathrm{O}_{5}+\mathrm{H}_{2} \mathrm{O} \rightarrow 2 \mathrm{HNO}_{3}$ & $*$ \\
\hline H13. & $\mathrm{ClONO}_{2}+\mathrm{H}_{2} \mathrm{O} \rightarrow \mathrm{HOCl}+\mathrm{HNO}_{3}$ & $*$ \\
\hline H14. & $\mathrm{BrONO}_{2}+\mathrm{H}_{2} \mathrm{O} \rightarrow \mathrm{HOBr}+\mathrm{HNO}_{3}$ & $*$ \\
\hline H15. & $\mathrm{ClONO}_{2}+\mathrm{HCl} \rightarrow \mathrm{Cl}_{2}+\mathrm{HNO}_{3}$ & $*$ \\
\hline H16. & $\mathrm{HOCl}+\mathrm{HCl} \rightarrow \mathrm{Cl}_{2}+\mathrm{H}_{2} \mathrm{O}$ & $*$ \\
\hline \multirow[t]{2}{*}{ H17. } & $\mathrm{HOBr}+\mathrm{HCl} \rightarrow \mathrm{BrCl}+\mathrm{H}_{2} \mathrm{O}$ & $*$ \\
\hline & \multicolumn{2}{|c|}{ Sea-salt aerosol reactions } \\
\hline HSSO. & $\mathrm{BrONO}_{2} \rightarrow 0.65 \mathrm{Br}_{2}+0.35 \mathrm{BrCl}$ & $\gamma=0.08$ \\
\hline HSS1. & $\mathrm{BrNO}_{2} \rightarrow 0.65 \mathrm{Br}_{2}+0.35 \mathrm{BrCl}$ & $\gamma=0.04$ \\
\hline HSS2. & $\mathrm{HOBr} \rightarrow 0.65 \mathrm{Br}_{2}+0.35 \mathrm{BrCl}$ & $\gamma=0.1$ \\
\hline HSS3. & $\mathrm{ClONO}_{2} \rightarrow \mathrm{Cl}_{2}$ & $\gamma=0.02$ \\
\hline HSS4. & $\mathrm{ClNO}_{2} \rightarrow \mathrm{Cl}_{2}$ & $\gamma=0.02$ \\
\hline HSS5. & $\mathrm{HOCl} \rightarrow \mathrm{Cl}_{2}$ & $\gamma=0.1$ \\
\hline HSS6. & $\mathrm{IONO}_{2} \rightarrow 0.5 \mathrm{IBr}+0.5 \mathrm{ICl}$ & $\gamma=0.01$ \\
\hline HSS7. & $\mathrm{INO}_{2} \rightarrow 0.5 \mathrm{IBr}+0.5 \mathrm{ICl}$ & $\gamma=0.02$ \\
\hline HSS8. & $\mathrm{HOI} \rightarrow 0.5 \mathrm{IBr}+0.5 \mathrm{ICl}$ & $\gamma=0.06$ \\
\hline
\end{tabular}

(*) As in Table A4 from Auxiliary Material in Kinnison et al. (2007)

Following McFiggans et al. (2000) we applied the free molecular transfer approximation for the uptake of gas-phase halogen species on sea-salt aerosols. That is, the transfer coefficient $\left(\mathrm{s}^{-1}\right)$ is given by

$$
K=\frac{\gamma}{4}<c>A
$$

where $\gamma$ is the uptake coefficient, $<\mathrm{c}>$ is the root-mean-square molecular speed, and A is the total available sea-salt surface area density $\left(\mathrm{cm}^{2} / \mathrm{cm}^{3}\right) .<\mathrm{c}>$ is given by:

$$
<c>=100 \sqrt{\frac{8 R T}{\pi M}}
$$

with $\mathrm{R}=8.3145 \mathrm{~J} \mathrm{~mol}^{-1} \mathrm{~K}^{-1}, \mathrm{~T}$ is the temperature and $\mathrm{M}$ the molecular mass of the species (in $\mathrm{Kg} \mathrm{mol}^{-1}$ ). The factor 100 converts to $\mathrm{cm} \mathrm{s}^{-1}$. 
Table 5. Henry's Law constants for relevant halogen species in CAM-Chem.

\begin{tabular}{llll}
\hline Species & $\mathrm{k}_{0}\left(\mathrm{M} \mathrm{atm}^{-1}\right)$ & $\mathrm{c}(\mathrm{K})$ & Reference \\
\hline $\mathrm{ClONO}_{2}$ & $1.0 \times 10^{6}$ & & $1, \mathrm{a}$ \\
$\mathrm{BrONO}_{2}$ & $1.0 \times 10^{6}$ & & $1, \mathrm{a}$ \\
$\mathrm{HCl}$ & $1.9 \times 10^{1}$ & 9000 & $1, \mathrm{~b}$ \\
$\mathrm{HOCl}$ & $9.3 \times 10^{2}$ & & $1, \mathrm{~b}$ \\
$\mathrm{HOBr}$ & $1.9 \times 10^{3}$ & & $1, \mathrm{c}$ \\
$\mathrm{HBr}$ & $7.2 \times 10^{-1}$ & 6100 & $1, \mathrm{~b}$ \\
$\mathrm{BrCl}$ & $7.0 \times 10^{-2}$ & 4000 & 3 \\
$\mathrm{IBr}$ & $2.4 \times 10^{1}$ & & 1 \\
$\mathrm{ICl}$ & $1.1 \times 10^{2}$ & & 1 \\
$\mathrm{BrNO}$ & $3.0 \times 10^{-1}$ & & 1 \\
$\mathrm{ClNO}_{2}$ & $4.0 \times 10^{-2}$ & & $1, \mathrm{c}$ \\
$\mathrm{HI}$ & $2.5 \times 10^{1}$ & 9800 & 3 \\
$\mathrm{HOI}$ & $4.5 \times 10^{2}$ & & 2 \\
\hline
\end{tabular}

Temperature dependent Henry's law constants are calculated as:

where

$$
k_{H}=k_{0} \times \exp \left[c\left(\frac{1}{T}-\frac{1}{T_{0}}\right)\right]
$$

$$
\begin{aligned}
& \mathrm{k}_{0}=\text { value of } \mathrm{k}_{\mathrm{H}} \text { at standard conditions } \\
& \mathrm{T}_{0}=298.15 \mathrm{~K}
\end{aligned}
$$

${ }^{1}$ Sander (1999)

${ }^{a}$ Virtually infinite solubility is represented in the model using a very large but arbitrary number.

${ }^{\mathrm{b}}$ Other values are also reported in the corresponding reference.

${ }^{c}$ Within the range of values given in the corresponding reference.

${ }^{2}$ Chemical mechanism of MECCA (Sander et al., 2011).

${ }^{3}$ Estimated. 
Table 6. Dry deposition velocities of relevant halogen species in CAM-Chem.

\begin{tabular}{lc}
\hline Species & Deposition velocity $\left(\mathbf{c m ~ s}^{-1}\right)$ \\
$\mathrm{HCl}, \mathrm{HBr}$ & 2.00 \\
$\mathrm{HOCl}, \mathrm{ClONO}$ & 1.00 \\
$\mathrm{HOBr}$ & 1.60 \\
$\mathrm{BrONO}$ & \\
$\mathrm{HI}$ & 0.50 \\
$\mathrm{I}_{2} \mathrm{O}_{2}$ & 1.00 \\
$\mathrm{HOI}, \mathrm{IONO}_{2}, \mathrm{NO}_{2}$ & 1.00 \\
\hline
\end{tabular}




\section{References}

Aranda, A., Le Bras, G., La Verdet, G., and Poulet, G.: The $\mathrm{BrO}+\mathrm{Ch} 3 \mathrm{O} 2$ reaction: Kinetics and role in the atmospheric ozone budget, Geophys. Res. Lett., 24, 22, doi:10.1029/97GL02686, 1997.

Atkinson, R., Baulch, D. L., Cox, R. A., Hampson, R. F., Kerr, J. A., Rossi, M. J., and Troe, J.: Evaluated Kinetic and Photochemical Data for Atmospheric Chemistry: Supplement VIII, Halogen Species Evaluation for Atmospheric Chemistry, J. Phys. Chem. Ref. Data 29, 167, 2000.

Atkinson, R., et al: Summary of evaluated kinetic and photochemical data for atmospheric chemistry: Web version April 2007, http://www.iupac-kinetic.ch.cam.ac.uk, $2007 \mathrm{a}$.

Atkinson, R., Baulch, D. L., Cox, R. A., Crowley, J. N., Hampson, R. F., Hynes, R. G., Jenkin, M. E., Rossi, M. J., and Troe, J.: Evaluated kinetic and photochemical data for atmospheric chemistry: Volume III - gas phase reactions of inorganic halogens, Atmos. Chem. Phys., 7, 981-1191, $2007 b$.

Boxe, C. S., and Saiz-Lopez, A.: Multiphase modeling of nitrate photochemistry in the quasi-liquid layer (QLL): implications for $\mathrm{NO}_{\mathrm{x}}$ release from the Arctic and coastal Antarctic snowpack, Atmos. Chem. Phys., 8, 4855-4864, 2008.

Chambers, R. M., Heard, A. C., and Wayne, R. P.: Inorganic gas-phase reactions of the nitrate radical: iodine + nitrate radical and iodine atom + nitrate radical, J. Phys. Chem., 96, 3321-3331, 1992.

Fickert, S., Adams, J.W., and Crowley, J. N.: Activation of $\mathrm{Br}_{2}$ and $\mathrm{BrCl}$ via uptake of HOBr onto aqueous salt solutions, J. Geophys. Res.-Atmos., 104, D19, 23719-23727, 1999.

Gómez Martín, J. C., Spietz, P., and Burrows, J. P.: Kinetic and Mechanistic Studies of the $\mathrm{I}_{2} / \mathrm{O}_{3}$ Photochemistry, J. Phys. Chem. A, 111, 306-320, 2007.

Kinnison, D. E., Brasseur, G. P., Walters, S., Garcia, R. R., Marsh, D. R., and Sassi, F., Harvey, V. L., Randall, C. E., Emmons, L., Lamarque, J. F., Hess, P., Orlando, J. J., Tie, X. X., Randel, W., Pan, L. L., Gettelman, A., Granier, C., Diehl, T., Niemeier, U., and Simmons, A. J.: Sensitivity of chemical tracers to meteorological parameters in the 
MOZART-3 chemical transport model, J. Geophys. Res. 112, D20302, doi:10.1029/2006JD007879, 2007.

McFiggans, G., Plane, J. M. C., Allan, B. J., Carpenter, L. J., Coe, H., and O'Dowd, C.: A modeling study of iodine chemistry in the marine boundary layer, J. Geophys. Res., 105(D11), 14, 371-14, 385, 2000.

National Institute of Standards and Technology (NIST), Chemical Kinetics Database on the Web, Standard Reference Database 17, Version 7.0 (Web Version), Release 1.5 http://kinetics.nist.gov/kinetics, 2010.

Read, K. A., Mahajan, A. S., Carpenter, L. J., Evans, M. J., Faria, B. V. E., Heard, D. E., Hopkins, J. R., Lee, J. D., Moller, S. J., Lewis, A. C., Mendes, L., McQuaid, J. B., Oetjen, H., Saiz-Lopez, A., Pilling, M. J., and Plane, J. M. C.: Extensive halogenmediated ozone destruction over the tropical Atlantic Ocean, Nature, 453, 1232-1235, 2008.

Saiz-Lopez, A., Mahajan, A. S., Salmon, R. A., Bauguitte, S. J.-B, Jones, A. E., Roscoe, H. K., and Plane, J. M. C.: Boundary Layer Halogens in Coastal Antarctica, Science, 317, 348-351, 2007.

Saiz-Lopez, A., Plane, J. M. C., Mahajan, A. S., Anderson, P. S., Bauguitte, S. J.-B., Jones, A. E., Roscoe, H. K., Salmon, R. A., Bloss, W. J., Lee, J. D., and Heard, D. E.: On the vertical distribution of boundary layer halogens over coastal Antarctica: implications for $\mathrm{O}_{3}, \mathrm{HO}_{\mathrm{x}}, \mathrm{NO}_{\mathrm{x}}$ and the $\mathrm{Hg}$ lifetime, Atmos. Chem. Phys., 8, 887-900, 2008.

Sander, R: Compilation of Henry's Law Constants for Inorganic and Organic Species of Potential Importance in Environmental Chemistry (Version 3) http://www.henryslaw.org, 1999.

Sander, R., et al.: The Chemical Mechanism of MECCA, KPP version: 2:2:1_rs5, MECCA version: 3:0. Part of the electronic supplement to the article "The atmospheric chemistry box model CAABA/MECCA-3.0", in Geosci. Model Dev., 373-380, 2011.

Sander, S. P., Friedl, R. R., Golden, D. M., Kurylo, M. J., Huie, R. E., Orkin, V. L, Moortgat, G. K., Ravishankara, A. R., Kolb, C. E., Molina, M. J., and Finlayson-Pitts, B. J.: Chemical Kinetics and Photochemical Data for Use in Atmospheric Studies Evaluation Number 14, JPL Publication 02-25, 2003. 
Sander, S. P., Friedl, R. R., Golden, D. M., Kurylo, M. J., Moortgat, G. K., KellerRudek, H., Wine, P. H., Ravishankara, A. R., Kolb, C. E., Molina, M. J., Finlayson-Pitts, B. J., Huie, R. E., and Orkin, V. L.: Chemical Kinetics and Photochemical Data for Use in Atmospheric Studies - Evaluation Number 15, JPL Publication 06-2, 2006.

von Glasow, R., Sander, R., Bott, A., and Crutzen, P. J.: Modelling halogen chemistry in the marine boundary layer 1. Cloud-free MBL, J. Geophys. Res., 107, 4341, doi:10.1029/2001JD000942, 2002.

von Glasow, R., and Crutzen, P. J.: Tropospheric Halogen Chemistry, Treatise on Geochemistry, 4, 1-67, 2007.

Warwick, N. J., Pyle, J. A., Carver, G. D., Yang, X., Savage, N. H., O’Connor, F. M., and Cox, R. A.: Global modelling of biogenic bromocarbons, J. Geophys. Res., 111, D24305, doi:10.1029/2006JD007264, 2006.

Yang, X., Cox, R. A., Warwick, N. J., Pyle, J. A., Carver, G. D., O’Connor, F. M., and Savage, N. H.: Tropospheric bromine chemistry and its impacts on ozone: A model study, J. Geophys. Res., 110, D23311, doi:10.1029/2005JD006244, 2005. 